\title{
Rationale and Design of the Carotid Plaque in Human for All Evaluations With Aggressive Rosuvastatin Therapy (CHALLENGER Trial)
} Evaluation by Magnetic Resonance Imaging

\author{
Katsumi Miyauchi, MD; Norihide Takaya, MD; Takahisa Hirose, MD*; Fuki Ikeda, MD*; \\ Ryuzo Kawamori, MD*; Hidenori Ohishi, MD**; Kensaku Yoshida, MD**; \\ Munetaka Yamamoto, MD**; Hajime Arai, MD**; Takao Urabe, MD"; \\ Nobutaka Hattori, MD ${ }^{\dagger}$; Michimasa Suzuki, MD ${ }^{\dagger+}$; Tadayuki Maehara, MD ${ }^{\dagger \dagger}$; \\ Kazuhiro Sase, MD $¥$; Thomas S. Hatsukami, MD $\$$, $\$$; \\ Chun Yuan, $\mathrm{PhD}^{\S}$; Hiroyuki Daida, $\mathrm{MD}$
}

\begin{abstract}
Background Intensive lipid-lowering therapy with statins reduces levels of low-density lipoprotein (LDL)cholesterol (C) and improves plaque volume and composition in patients with cardiovascular disease. Furthermore, rosuvastatin ameliorated carotid stenosis in the ASTEROID study, and altered the composition of plaques in a predominantly Caucasian study population in the ORION study. However, it is not known whether statin therapy achieves similar quantitative improvement in carotid artery plaque in other ethnic groups.

Methods and Results Fifty patients with hypercholesterolemia (LDL-C $\geq 120 \mathrm{mg} / \mathrm{dl}$ ) and a maximum carotid intima-media thickness $\geq 1.8 \mathrm{~mm}$ will be enrolled and treated with rosuvastatin at a dose of $5 \mathrm{mg} / \mathrm{day}$ for 96 weeks. The primary endpoints will be the percent change of carotid plaque volume and the change in plaque composition after 96 weeks of treatment, as evaluated by magnetic resonance imaging.

Conclusions The CHALLENGER study will provide a noninvasive assessment of the changes in carotid plaque volume and composition achieved by reduction of LDL levels in Japanese patients with carotid stenosis on long-term rosuvastatin therapy. (Circ J 2009; 73: 111-115)
\end{abstract}

Key Words: Carotid intima-media thickness; Carotid plaque; High-resolution magnetic resonance imaging; Plaque stability; Rosuvastatin

$\mathbf{C}$ erebrovascular accidents are the third leading cause of death in Japan. Although the incidence of cerebral hemorrhage has decreased markedly since approximately 1990, that of cerebral infarction has increased. In addition, there has been a decrease in the incidence of lacunar infarction and an increase of thrombotic infarction secondary to atherosclerotic plaque, as well as an increase in cardioembolic infarction. Therefore, the incidence and pattern of stroke in Japan has become similar to that in Western countries!

Hypercholesterolemia is a major risk factor for atherosclerosis and is closely related to the development of ischemic heart disease. Several large-scale trials have demonstrated that statins with a potent lipid-lowering

(Received February 20, 2008; revised manuscript received July 24, 2008; accepted August 17, 2008; released online December 4, 2008) Departments of Cardiovascular Medicine, *Medicine, Metabolism \& Endocrinology, ${ }^{* *}$ Neurosurgery, ${ }^{\dagger}$ Neurology, ${ }^{\dagger}$ Radiology, ${ }^{\star}$ Clinical Phamacology, Juntendo University School of Medicine, Tokyo, Japan, Departments of $\$$ Surgery, $\$$ Radiology, University of Washington and \$\$A Puget Sound Health Care System, Seattle, WA, USA

Mailing address: Hiroyuki Daida, MD, Department of Cardiovascular Medicine, Juntendo University School of Medicine, 3-1-3 Hongo, Bunkyo-ku, Tokyo 113-0033, Japan. E-mail: daida@med. juntendo.ac.jp

All rights are reserved to the Japanese Circulation Society. For permissions, please e-mail: cj@j-circ.or.jp action have a preventive effect on the recurrence of myocardial infarction ${ }^{2-6}$ and further clinical trials to investigate the efficacy of statins on coronary plaque are currently ongoing?, 8

When patients who have coronary artery disease are treated with statins, the decrease in the level of low-density lipoprotein (LDL)-cholesterol (C) is positively correlated with a reduction in plaque size, and plaque regression is observed when the patient achieves LDL-C $=78 \mathrm{mg} / \mathrm{dl}$ ? In our previous study (Early Statin Treatment in Patients with Acute Coronary Syndrome: ESTABLISH), aggressive lipid-lowering therapy with atorvastatin for 6 months decreased LDL-C by $41.7 \%$ and reduced the coronary plaque volume by $13.1 \%$ in patients with acute coronary syndrome. The percent change in plaque volume showed a positive correlation with the percent reduction of LDL$\mathrm{C}$, even in patients with low baseline LDL-C levels! ${ }^{10}$ Another study (A Study To evaluate the Effect of Rosuvastatin On intravascular ultrasound-Derived coronary atheroma burden: ASTEROID), demonstrated that aggressive treatment with rosuvastatin for 2 years could decrease the LDL-C level to $60.8 \mathrm{mg} / \mathrm{dl}$ from a baseline of $130.4 \mathrm{mg} / \mathrm{dl}$ in patients with coronary heart disease confirmed by coronary angiography, resulting in a significant reduction of plaques volume by $6.8 \%$ (vs baseline) $!^{1}$ These results indicate that there is a positive correlation between reduc- 
ing the LDL-C level and either inhibiting coronary plaque progression or inducing plaque regression.

Statins are also effective for preventing cerebral infarction. A meta-analysis has indicated that there is a positive correlation between prevention of cerebral infarction and reduction of LDL-C level, and it has been also shown that an increase in the carotid intima-media thickness (IMT) is strongly correlated with the incidence of cerebral infarction. The concept of plaque stabilization is based on the finding that a $34 \%$ decrease in LDL-C level was associated with changes in the lipid content and inflammatory activity of plaques from patients treated with pravastatin for 3 months compared with controls not receiving pravastatin!2 Recently, high-resolution magnetic resonance imaging (MRI) has been used for evaluating carotid plaque and satisfactory results have been obtained, with respect to assessing changes in plaque volume and composition! ${ }^{13-17}$

Rosuvastatin has a potent lipid-lowering action and has been approved in more than 80 countries worldwide $!^{3}$ The ORION (Outcome of Rosuvastatin treatment on carotid artery atheroma: a magnetic resonance Imaging ObservatioN) study suggested a relationship between marked reduction of LDL-C and inhibition of the progression of atherosclerosis in patients with moderate hypercholesterolemia and carotid stenosis receiving rosuvastatin at $5 \mathrm{mg} /$ day or $40 \mathrm{mg} /$ day for 2 years, and also showed that a marked reduction in LDL-C was associated with a decrease in the size of the lipid-rich necrotic core (LRNC) of plaques and increased fibrous tissue in the carotid plaques of American patients $!^{14,15}$ However, the effects of rosuvastatin on carotid plaque in Japanese patients have not been clarified. The CHALLENGER trial will determine whether rosuvastatin can alter the composition and volume of carotid plaques in Japanese patients. It was considered that rosuvastatin was likely to contribute to qualitative and quantitative improvement of carotid plaque in Japanese patients. We therefore decided to perform a study of lipid-lowering therapy with rosuvastatin $(5 \mathrm{mg}$ /day for 96 weeks) in hypercholesterolemic patients who had a maximum carotid IMT $>1.8 \mathrm{~mm}$ to assess the changes of carotid plaque volume and composition by MRI. In this study, the coronary plaques will be measured by computed tomography (CT) angiography (which is a well-established technique) in subjects who are found to have coronary stenosis. We will also assess qualitative and quantitative changes of plaques over time in relation to changes in the LDL-C level.

\section{Methods}

Study Design

Single-group open trial.

\section{Study Population}

Patients who have hypercholesterolemia and a maximum IMT $\geq 1.8 \mathrm{~mm}$ as measured by ultrasound.

\section{Inclusion Criteria}

(1) Patients giving written consent after being provided with sufficient explanation about participation in this clinical trial.

(2) Patients aged 20 years or older at the time of giving consent (no limitations on gender or hospitalization status).
(3) Patients with hypercholesterolemia and a maxIMT $\geq 1.8 \mathrm{~mm}$ as measured by ultrasound.

(4) Patients with a serum LDL-C level $>120 \mathrm{mg} / \mathrm{dl}$, or patients who have both carotid stenosis and coronary stenosis with a serum LDL-C level $>100 \mathrm{mg} / \mathrm{dl}$.

\section{Exclusion Criteria}

(1) Patients currently receiving other lipid-lowering drugs apart from the test drug.

(2) Patients who have received any statin within 3 months before the observation period.

(3) Patients with severe carotid stenosis and marked calcification.

(4) Patients with a fasting triglyceride (TG) level $>400 \mathrm{mg} / \mathrm{dl}$.

(5) Patients with uncontrolled hypertension (diastolic blood pressure $\geq 100 \mathrm{mmHg}$ or systolic blood pressure $\geq 200 \mathrm{mmHg}$ throughout the observation period).

(6) Patients with familial hypercholesterolemia that cannot be controlled by statin therapy.

(7) Patients with secondary hypercholesterolemia because of thyroid dysfunction, Cushing's syndrome, nephrotic syndrome, systemic lupus erythematosus, etc.

(8) Patients receiving LDL apheresis.

(9) Patients on treatment with cyclosporine.

(10) Patients with renal dysfunction or on hemodialysis.

(11) Patients with a history of severe adverse effects or allergy to statins.

(12) Patients with confirmed malignancy, possible malignancy, or a history of malignancy.

(13) Patients with active liver disease or hepatic dysfunction (indicated by alanine aminotransferase (ALT), aspartate aminotransferase (AST), and/or alkaline phosphatase (ALP) levels more than 2.5-fold the upper limit of normal, or a total bilirubin $>3.0 \mathrm{mg} / \mathrm{dl}$ ).

(14) Patients with a serum creatinine $>1.5 \mathrm{mg} / \mathrm{dl}$.

(15) Patients with a serum creatine kinase (CK) level at least 3-fold the upper limit of normal.

(16) Patients with contraindications to MRI.

(17) Inadequate MRI images.

(18) Patients who have had carotid endarterectomy or radiation therapy in the region of the carotid artery.

(19) Patients with intraplaque hemorrhage.

(20) Patients who are ineligible for any other reason in the opinion of the investigator.

\section{Calculation of the Study Population}

We assumed that the percent change in LDL-C would be $40 \%$, that of \%LRNC would be -1.5 , and the standard deviation would be 3.0 during 96 weeks of rosuvastatin therapy at $5 \mathrm{mg} /$ day, because the ORION study showed that the \%LRNC was reduced by 1.33 and LDL-C was lowered by $40 \mathrm{mg} / \mathrm{dl}$ after 2 years of rosuvastatin treatment. We also assumed that a statistically meaningful change of $\%$ LRNC would be -1.5 . Accordingly, we calculated that 50 participants were needed for an alpha level of 5\% and $90 \%$ power of test, as well as considering possible dropouts.

\section{Study Medication and Treatment Period}

Rosuvastatin will be administered for 96 weeks at a dose of $5 \mathrm{mg} /$ day. If treatment for 4 weeks fails to reduce the LDL-C level below $120 \mathrm{mg} / \mathrm{dl}$, the dose can be increased up to $20 \mathrm{mg}$ /day. 


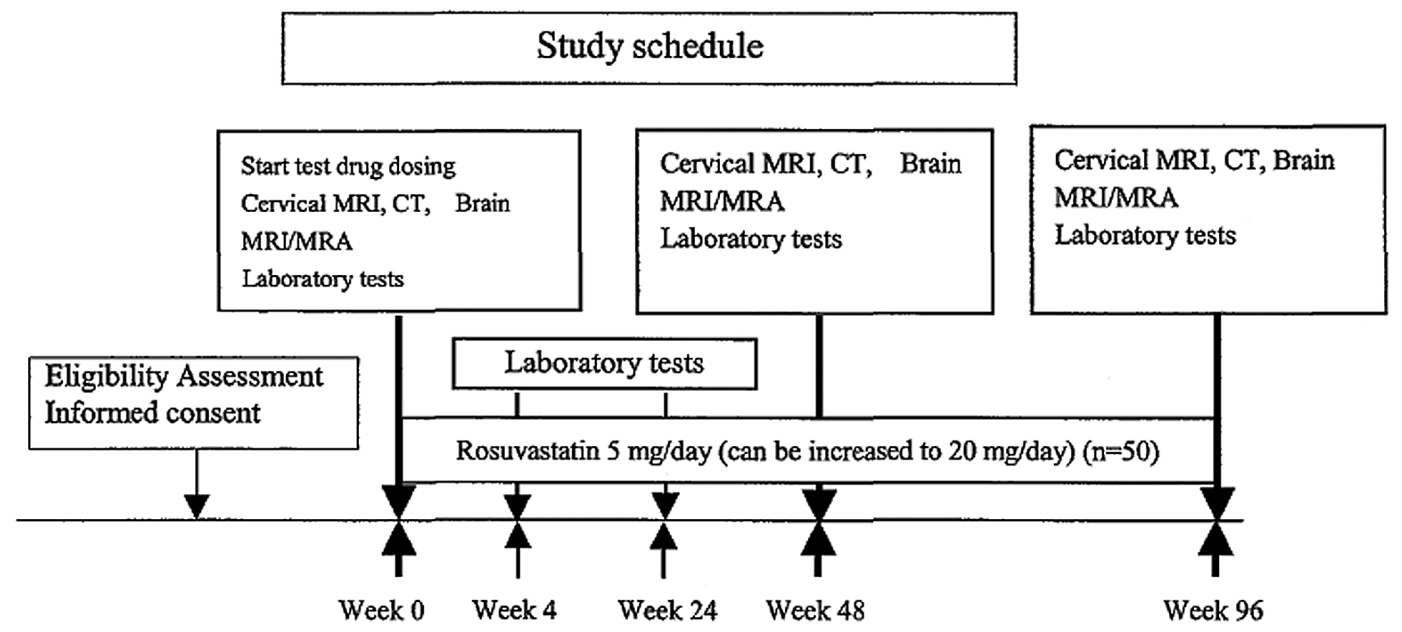

Fig 1. Outline of the study schedule. CT, computed tomography; MRA, magnetic resonance angiography; MRI, magnetic resonance imaging.

Table 1 Study Schedule

\begin{tabular}{|c|c|c|c|c|c|}
\hline & $\begin{array}{c}\text { Visit } 1 \\
\text { Week } 0\end{array}$ & \multirow{2}{*}{$\begin{array}{l}\text { Visit } 2 \\
\text { Week } 4\end{array}$} & \multirow{2}{*}{$\begin{array}{c}\text { Visit } 3 \\
\text { Week } 24\end{array}$} & \multirow{2}{*}{$\begin{array}{c}\text { Visit } 4 \\
\text { Week } 48\end{array}$} & \multirow{2}{*}{$\begin{array}{c}\text { Visit } 5 \\
\text { Week } 96 \\
\text { Completion }\end{array}$} \\
\hline & Screening & & & & \\
\hline Patient characteristics & 0 & & & & \\
\hline Informed consent & 0 & & & & \\
\hline Waist, height, body weight & 0 & & & & 0 \\
\hline Inclusion/exclusion criteria & 0 & & & & \\
\hline Carotid ultrasound & 0 & & & & \\
\hline Cervical MRI/MRA & 0 & & & 0 & 0 \\
\hline CT angiography* & $(\bigcirc)$ & & & (○) & (○) \\
\hline Brain MRI/MRA** & (O) & & & (O) & (0) \\
\hline Fasting serum lipids & 0 & 0 & 0 & 0 & 0 \\
\hline$h s-C R P$ & 0 & 0 & 0 & 0 & 0 \\
\hline Fasting blood glucose, insulin, and $\mathrm{HbAlc}$ & 0 & 0 & 0 & 0 & 0 \\
\hline Laboratory tests ${ }^{\dagger}$ & $(\bigcirc)$ & $(\bigcirc)$ & $(\bigcirc)$ & $(\bigcirc)$ & $(\bigcirc)$ \\
\hline Concomitant drugs & 0 & 0 & 0 & 0 & 0 \\
\hline Compliance with therapy & 0 & 0 & 0 & 0 & 0 \\
\hline Adverse events & 0 & 0 & 0 & 0 & $\mathrm{O}$ \\
\hline
\end{tabular}

*CT angiography is only performed when coronary stenosis is suspected on the basis of other tests.

**Brain MRI/MRA will be performed when a subject has a suspected cerebrovascular accident and is in a suitable condition for these examinations.

Laboratory tests will measure standard items.

MRI, magnetic resonance imaging; MRA, magnetic resonance angiography; $C T$, computed tomography; hs-CRP, high-sensitivity $C$-reactive protein; $\mathrm{Hb}$, hemoglobin.

\section{Items Investigated}

Following data are collected as shown in Fig1 and Table1.

(1) Patient's characteristics: initials (first name, family name), sex, birth date, height, weight, waist circumference, blood pressure, heart rate, complications, and past history.

(2) Concomitant drug therapy: confirm whether prohibited concomitant drugs are being used.

(3) Confirm compliance with therapy at each interview: (i) $100 \%$, (ii) $75-100 \%$, (iii) $50-75 \%$, (iv) $\leq 50 \%$.

(4) Measurement of plaque: carotid artery ultrasound, MRI, CT angiography.

(5) Other imaging procedures: MRI and magnetic resonance angiography (MRA) of the head.

(6) Serum lipids and inflammatory markers: LDL-C, high-density lipoprotein (HDL)-C, TG, high-sensitivity C-reactive protein (hs-CRP).

(7) Glucose metabolism: fasting blood glucose, insulin, serum hemoglobin A1c (HbA1c).

(8) Clinical examination and urinalysis: perform routine examinations for diagnosis and treatment. As well as measuring serum creatinine, urinalysis will be done at visit 1 and visit 5 whenever possible.

\section{Study Endpoints}

Primary Endpoints The percent change in carotid plaque volume and the changes in carotid plaque composition in week 96.

Secondary Endpoints There are 5 secondary endpoints.

(1) Percent change in carotid plaque volume and qualitative plaque change in week 48 .

(2) Percent changes in LDL-C and HDL-C, the LDLC/HDL-C ratio, hs-CRP and glomerular filtration rate (GFR).

(3) Relationship between changes in LDL-C and 
plaque volume, between changes in HDL-C and plaque volume, and between changes in hs-CRP and plaque volume.

(4) Details and incidence of major adverse cardiac and cerebrovascular events.

(5) Relationship between hyperinsulinemia and carotid plaque.

\section{Target Plaque}

When the target plaque in the carotid artery is assessed by MRI, a region of plaque $20 \mathrm{~mm}$ in length, including the index lesion, will be imaged.

\section{MRI Protocol and Review}

All patients will undergo MRI at Juntendo University School of Medicine, using a 1.5-T Siemens Scanner (Magnetom Avanto, NY, USA) with a phased-array surface coil (Machnet, The Netherlands). A standardized protocol ${ }^{18}$ will be used to obtain 4 different types of images of the index carotid artery in the transverse plane: T1-weighted images (T1W), proton density-weighted images (PDW), T2-weighted images (T2W), and 3-dimensional time-offlight MR angiography. Fat suppression will be used for the $\mathrm{T} 1 \mathrm{~W}, \mathrm{PDW}$, and $\mathrm{T} 2 \mathrm{~W}$ images to reduce signals from subcutaneous fat and improve assessment of the total vessel area. Gadolinium-enhanced T1W will be obtained if the patient agrees.

For area measurement, the lumen and outer vessel wall boundaries (total vessel area) will be traced using a custom-designed image analysis tool (CASCADE) 19 Plaque components, such as the necrotic core, calcification, loose matrix, and fibrous tissue, will be analyzed by automated segmentation of the plaques as mean area for the artery and mean $\%$ of wall area on MRI ${ }^{20}$

\section{Statistical Analysis}

The plaque volume and the MRI value measured at the start of the study will be used as the baseline values for calculation of the percent changes in week 96, as well as the 2 -sided $95 \%$ confidence interval. Next, the 1-sample t-test and the Wilcoxon signed rank test will be used to determine that the percent change in each plaque is not 0 . The 2-sided level of significance level will be set at $\mathrm{P}<0.05$.

In addition, the relationship between the percent change in plaque volume and the various lipids or inflammation markers will be investigated in an exploratory manner for each dose of the test drug.

\section{Discontinuation}

(1) A patient requests to withdraw from the study during the study period.

(2) It is considered difficult to continue the study treatment because of the occurrence of adverse events.

(3) Serum CK exceeds $1,000 \mathrm{U} / \mathrm{L}$.

(4) The serum ALT, AST, or ALP level is persistently $\geq 3$ times the upper limit of normal.

(5) Serum creatinine increases to $\geq 1.5 \mathrm{mg} / \mathrm{dl}$.

(6) No lipid-lowering effect is observed after administration of the study medication.

(7) Performance of assessment by MRI is considered impossible.

(8) A significant protocol violation occurs.

(9) The investigator deems it necessary to discontinue the study treatment.

\section{Study Period}

The study is planned to run from July 2007 to July 2010.

\section{Publication of the Results and Protection of Privacy}

The study results will be reported to an academic society and published in a medical journal. However, individual patient information will be masked and the privacy of subjects will be protected.

\section{Study Organization}

Principal Investigator Hiroyuki Daida, Department of Cardiovascular Medicine, Juntendo University School of Medicine.

Steering Committee Katsumi Miyauchi, Department of Cardiovascular Medicine, Juntendo University School of Medicine; Norihide Takaya, Department of Cardiovascular Medicine, Juntendo University School of Medicine; Takahisa Hirose, Metabolism \& Endocrinology, Juntendo University School of Medicine; Hidenori Ohishi, Department of Neurosurgery, Juntendo University School of Medicine; Takao Urabe, Department of Neurology, Juntendo University School of Medicine; Michimasa Suzuki, Department of Radiology, Juntendo University School of Medicine.

\section{Discussion}

Hypercholesterolemia is a major risk factor for cardiovascular and cerebrovascular (CCV) events, and a reduction in the level of LDL-C reduces both the risk of such events and the disease-related mortality. In view of this association, there are guidelines for the target LDL-C levels for patients at risk of developing CCV events. In the National Cholesterol Education Program Adult Treatment Panel III guidelines, patients with a high absolute risk of a CCV event have the most stringent target, which requires intensive LDL-C lowering therapy?1 Japan Atherosclerosis Society Guidelines for Prevention of Atherosclerotic Cardiovascular Diseases also recommend aggressive lipid-lowering therapy for patients with a high risk of CCV events? 22

Statin therapy is effective for both primary and secondary prevention of myocardial infarction, stroke, and other cardiovascular events $2,23,24$ Several large-scale clinical trials have demonstrated that statins can prevent the recurrence of ischemic heart disease, ${ }^{2-4}$ and can also prevent the recurrence of stroke5,6 The major effect of statins is to reduce LDL-C, which remains the primary target of lipidlowering therapy for improving the risk of CCV, according to the abovementioned guidelines. Statins are also known to affect other lipids, such as HDL-C and TG, although these effects vary in magnitude among the available agents and also depend on patient characteristics? $2,3,5,6,25$

Rosuvastatin is a statin with a strong lipid-lowering action. Several large-scale trials have shown that it is significantly more effective at reducing TC, LDL-C, and TG (while increasing HDL-C) than other statins ${ }^{26}$ The ASTEROID study showed that intensive rosuvastatin therapy $(40 \mathrm{mg} /$ day) caused a decrease in the volume of coronary artery atheroma! ${ }^{1}$ In addition, the METEOR (Measuring Effect on Intima Media Thickness: an Evaluation Of Rosuvastatin) study showed significant reductions in the rate of progression of maximum carotid IMT over 2 years vs placebo? 
In addition to those studies, the ORION study showed that treatment with rosuvastatin $(5 \mathrm{mg} /$ day $)$ for 2 years led to a reduction in LDL-C of $40 \%$ and regression of \%LRNC of $1.33 \% ! 4$ An increase in carotid IMT is known to be a strong predictor of the development of carotid plaque and stenosis, and is clearly associated with both the occurrence of stroke and the cholesterol level. CHALLENGER will use MRI to assess the effects of rosuvastatin on the volume and composition of carotid plaques in patients who have high-risk atherosclerosis with a max-IMT of more than $1.8 \mathrm{~mm}$. The quantitative regression and qualitative changes in carotid plaque will be assessed in patients receiving rosuvastatin at $5 \mathrm{mg} /$ day for 2 years.

CHALLENGER was designed as an open study (rosuvastatin treatment only) because the subjects are patients with a high risk of atherosclerotic complications who have a max-IMT of more than $1.8 \mathrm{~mm}$, so this design was chosen for safety and also because the clinical benefit of rosuvastatin for carotid plaque has already been established by the ORION study. CHALLENGER will provide useful data about the quantitative and qualitative changes in carotid plaque in Japanese patients with carotid stenosis receiving rosuvastatin therapy.

\section{References}

1. Kiyohara Y. Epidemiology of myocardial and cerebral infarction. Cardioangiology 2006; 59: 417-423.

2. Scandinavian Simvastatin Survival Study Group. Randomised trial of cholesterol lowering in 4,444 patients with coronary heart disease: The Scandinavian Simvastatin Survival Study (4S). Lancet 1994; 344: $1383-1389$.

3. Chau J, Cheung BM, McGhee SM, Lauder IJ, Lau CP, Kumana CR. Cost-effectiveness analysis of applying the Cholesterol and Recurrent Events (CARE) study protocol in Hong Kong. Hong Kong Med J 2001; 7: 360-368.

4. Keech A, Colquhoun D, Best J, Kirby A, Simes RJ, Hunt D, et al. Secondary prevention of cardiovascular events with long-term pravastatin in patients with diabetes or impaired fasting glucose: Results from the LIPID trial. Diabetes Care 2003; 26: 2713 2721.

5. Matsumoto M. Carotid atherosclerosis and lipid-lowering treatment: Assessment with ultrasonography. Cardioangiology 2005; 57: $42-49$.

6. Amarenco P, Labreuche J, Lavallee P, Touboul PJ. Statins in stroke prevention and carotid atherosclerosis: Systematic review and upto-date meta-analysis. Stroke 2004; 35: 2902-2909.

7. Takayama T, Hiro T, Yamagishi M, Daida H, Saito S, Yamaguchi $\mathrm{T}$, et al. Rationale and design for a study using intravascular ultrasound to evaluate effects of rosuvastatin on coronary artery atheroma in Japanese subjects: COSMOS study (Coronary atherosclerosis study measuring effects of rosuvastatin using intravascular ultrasound in Japanese patients. Circ J 2007; 71: 271-275.

8. Miyauchi K, Kiumura T, Morimoto T, Nakagawa Y, Yamagishi M, Ozaki Y, et al. Japan Assessment of pitavastatin and atprvastatin in acute coronay syndrome (JAPAN-ACS): Rationale and Design. Circ J 2006; 70: 1624-1628.

9. Birgelen C, Hartmann M, Mintz GS, Baumgart D, Schmermund A, Erbel R. Relation between progression and regression of atherosclerotic left main coronary artery disease and serum cholesterol levels as assessed with serial long-term (12 months) follow-up intravascular ultrasound. Circulation 2003; 108: 2757-2762.

10. Okazaki S, Yokoyama T, Miyauchi K, Shimada K, Kurata T, Sato $\mathrm{H}$, et al. Early statin treatment in patients with acute coronary syndrome: Demonstration of the beneficial effect on atherosclerotic lesions by serial volumetric intravascular ultrasound analysis during half a year after coronary events: The ESTABLISH study. Circulation 2004; 110: $1061-1068$.

11. Nissen SE, Nicholls SJ, Sipahi I, Libby P, Raichlen JS, Ballantyne $\mathrm{CM}$, et al. Effect of very high-intensity statin therapy on regression of coronary atherosclerosis: The ASTERID trial. JAMA 2006; 295: $1556-1565$.

12. Crisby M, Nordin-Fredriksson G, Shah PK, Yano J, Zhu J, Nilsson J. Pravastatin treatment increases collagen content and decreases lipid content, inflammation, metalloproteinases, and cell death in human carotid plaques: Implications for plaque stabilization. Circulation 2001; 103: 926-933.

13. Chu B, Hatsukami TS, Polissar NL, Zhao XQ, Kraiss LW, Parker $\mathrm{DL}$, et al. Determination of carotid artery atherosclerotic lesion type and distribution in hypercholesterolemic patients with moderate carotid stenosis using noninvasive magnetic resonance imaging. Stroke 2004; 35: 2444-2448.

14. Underhill HR, Hatsukami TS, Raichlen JS, Waterton J, Liu F, Kerwin WS, et al. Morphology-enhanced probabilistic plaque segmentation identifies regression of the lipid-rich portion of carotid plaques after 2 years of rosuvastatin therapy. Circulation 2006; 114: $377-378$.

15. Underhill HR, Yuan C, Zhao XQ, Kraiss LW, Parker DL, Saam T, et al. Effect of rosuvastatin therapy on carotid plaque morphology and composition in moderately hypercholesterolemic patients: A high-resolution magnetic resonance imaging trial. Am Heart J 2007; 155: $584 \mathrm{e} 1-584 \mathrm{e} 8$.

16. Watanabe K, Sugiyama S, Kugiyama K, Honda O, Fukushima H, Koga $\mathrm{H}$, et al. Atherosclerosis evaluation by ultrasound: Stabilization of carotid atheroma assessed by quantitative ultrasound analysis in nonhypercholesterolemic patients with coronary artery disease. $J$ Am Coll Cardiol 2005; 46: 2022-2030.

17. Takaya N, Cai J, Ferguson MS, Yarnykh VL, Chu B, Saam T, et al. Intra- and interreader reproducibility of magnetic resonance imaging for quantifying the lipid-rich necrotic core is improved with gadolinium contrast enhancement. J Magn Reson Imaging 2006; 24: $203-210$.

18. Saam T, Hatsukami TS, Yarnykh VL, Hayes CE, Underhill H, Chu $\mathrm{B}$, et al. Reader and platform reproducibility for quantitative assessment of carotid atherosclerotic plaque using 1.5T Siemens, Philips, and General Electric scanners. J Magn Reson Imaging 2007; 26: $344-352$.

19. Kerwin WS, Han C, Chu B. A quantitative vascular analysis system for evaluation of atherosclerotic lesions by MRI. In: Proceedings of the 4th International Conference on Medical Image Computing and Computer-Assisted Intervention (MICCAI), Utrecht, The Netherlands, 14-17 October 2001. Lecture Notes in Computing Science 2208 edn. Berlin: Springer; 2001; 786-794.

20. Liu F, Xu D, Ferguson MS, Chu B, Saam T, Takaya N, et al. Automated in vivo segmentation of carotid plaque MRI with Morphology-Enhanced probability maps Magn Reson Med 2006; 55: 659668.

21. National Cholesterol Education Program (NECP) Expert Panel on Detection, Evaluation, and Treatment of High Blood Cholesterol in Adults (Adult Treatment Panel III). Third Report of the National Cholesterol Education Program (NECP) Expert Panel on Detection, Evaluation, and Treatment of High Blood Cholesterol in Adults (adult treatment panel III) final report. Circulation 2002; 106: 3143 3421.

22. Teramoto T, Sasaki J, Ueshima H, Egusa G, Kinoshita M, Shimamoto K, et al. Executive Summary of Japan Atherosclerosis Society (JAS) Guideline for Diagnosis and Prevention of Atherosclerotic Cardiovascular Diseases for Japanese. J Atheroscler Thromb 2007; 14: 45-50.

23. Shepherd J, Cobbe SM, Ford I, Isles CG, Lorimer AR, MacFarlane PW, et al. Prevention of coronary heart disease with pravastatin in men with hypercholesterolemia: West of Scotland Coronary Prevention Study Group. N Engl J Med 1995; 333: 1301-1307.

24. Downs JR, Clearfield M, Weis S, Whitney E, Shapiro DR, Beere PA, et al. Primary prevention of acute coronary events with lovastatin in men and women with average cholesterol levels: Results of AFCAPS/TexCAPS. JAMA 1998; 279: 1615-1622.

25. Knopp RH. Drug treatment of lipid disorders. N Engl J Med 1999; 34: $498-511$.

26. Schuster H. The GALAXY program: An update on studies investigating efficacy and tolerability of rosuvastatin for reducing cardiovascular risk. Expert Rev Cardiovasc Ther 2007; 5: 177-192.

27. Crouse JR III, Raichlen JS, Riley WA, Evans GW, Palmer MK, O'Leary DH, et al. Effect of rosuvastatin on progression of carotid intima-media thickness in low-risk individuals with subclinical atherosclerosis. JAMA 2007; 297: 1344-1353. 